\title{
STARTING MECHANICAL THROMBECTOMY SERVICE DURING COVID-19 PANDEMIC: OUR EARLY INSTITUTION EXPERIENCE.
}

\author{
Ezamin Abdul Rahim ${ }^{1}$, Ahmad Sobri Muda ${ }^{1}$, Mohd Naim Mohd Yaakob ${ }^{1}$, Mohd Fandi Al Khafiz Kamis ${ }^{1}$, \\ Aizad Azahar ${ }^{2}$, Chong Kok $\mathrm{Wah}^{2}$, Chia Peck Kee ${ }^{3}$, Hamidon Basri ${ }^{3}$, Iskasymar Itam @ Ismail ${ }^{4}$, \\ Muhammad Mohd Isa ${ }^{5}$

\begin{abstract}
${ }^{1}$ Department of Imaging, Faculty of Medicine and Health Sciences, Universiti Putra Malaysia, 43600, Serdang, Selangor, Malaysia ${ }^{2}$ Department of Anaesthesiology, Faculty of Medicine and Health Sciences, Universiti Putra Malaysia, 43600, Serdang, Selangor, Malaysia ${ }^{3}$ Department of Neurology, Faculty of Medicine and Health Sciences, Universiti Putra Malaysia, 43600, Serdang, Selangor, Malaysia ${ }^{4}$ Regional Emergency Stroke Quick (RESQ) Response Unit, Hospital Pengajar Universiti Putra Malaysia, 43600, Serdang, Selangor, Malaysia

${ }^{5}$ Director, Hospital Pengajar Universiti Putra Malaysia, 43600, Serdang, Selangor, Malaysia
\end{abstract}

*Correspondence author: Dr Ezamin Abdul Rahim ${ }^{1}$. Department of Imaging, Faculty of Medicine and Health Sciences, Universiti Putra Malaysia, 43600, Serdang, Selangor, Malaysia. E mail: drezahar@gmail.com, Tel: +60196846933

DOI: https://doi.org/10.32896/cvns.v2n1.18-21

Published: 30.09 .2020

Keywords: Covid-19, Severe acute respiratory syndrome coronavirus 2 (SARS-CoV-2), acute stroke, mechanical thrombectomy, healthcare workers (HCW)

As we are writing this manuscript in early May 2020, we are still living in COVID-19 (also known as severe acute respiratory syndrome coronavirus 2 (SARS-CoV-2)) pandemic era (1). Malaysia is one of the many countries affected by this global pandemic.

To prevent the spread of COVID-19, National Health Institutions all over the world imposed mandatory usage of Personal protective equipments (PPE) to protect health care workers $(\mathrm{HCW})$ when dealing with high risk patients and COVID-19 positive patients ${ }^{2}$. However, the PPEs are finite and episodic shortages of PPEs are common all over the world ${ }^{2,3}$.

During this challenging time, University Putra Malaysia Teaching Hospital (HPUPM) has leapt forward to offer optimum stroke care to the community. The acute stroke patients will be stratified for eligibility for mechanical thrombectomy and thrombolysis treatment in our institution. The criteria used is dependant on institutional practice. At the height of the pandemic, it is difficult to ascertain which patient had contracted COVID-19. Alarming evidence emerged suggesting that one of the central nervous system (CNS) manifestation of COVID-19 is acute stroke. The patient may be asymptomatic or exhibiting COVID-19 symptoms ${ }^{4}$. There are also reports suggesting acute stroke in young and middle-aged adults as a sequela of COVID-19 4 . This manuscript aims to share our experience in starting the mechanical thrombectomy service for acute stroke at the height of COVID-19 pandemic.

Our hospital was launched amid the pandemic, in early 2020. We have a dedicated Emergency Unit catering for stroke named as Regional Emergency Stroke Quick (RESQ) Response Unit. During the current pandemic era, all hospital admissions including the ones via RESQ will be vetted by a special task force in order to assist with the COVID-19 protocol via a special platform so as not to jeopardise the door to needle time.

To reduce staff exposure to COVID-19; all patients are screened for signs and symptoms of COVID- $19^{5}$. Declaration forms are given to the patient or next of kin to be filled (Figure 1). This form is formulated based on Infectious Disease Prevention and Control Act 1988-Malaysia ${ }^{6}$ and is a prerequisite for admission. Its main purpose is to notify the HCW about the current status of the patient, to identify and predict which patients are at high risk of COVID-19 infection. A rapid test is performed (Sars-COVID-2 Antibody Test- Lateral Flow Method, Wondfo $^{\circledR}$ ) which is complementary to the reverse-transcription polymerase chain reaction (RT-PCR) test. A throat and nasal swab for RT-PCR tests is performed in all patients immediately after rapid blood test procedure. This test is mandatory for every patient. The result will be traced the day after (RT-PCR) swab was taken. If there is suspicion of COVID-19, the patient will be transferred to the nearest COVID-19 hospital for further management.

Team members evaluating code red patients should be kept to a minimum and the attending $\mathrm{HCW}$ will need to wear full PPE which includes full-body protection coverall suits, N95 mask, eye protection and double-layer gloves for easier doffing (Figure 2). This "enhanced" full PPE helps to alleviate any aerosolized events such as coughing, sneezing, CPR, and intubation ${ }^{2}$. The full PPE gives a strong perception of protection among the $\mathrm{HCW}$ and consequently will boost $\mathrm{HCW}$ morale. However the use of full PPE is not without problems. This coverall protective suit is usually a "nonbreathable" type (Figure 2). This kind of coverall protective suit will hamper heat and moisture transfer. The HCW wearing full PPE will experience heat stress, body irritation, discomfort and even may restrict movement. Wearing eye 
protection such as goggle and face shield will induce fogging, thus reducing the HCW vision. Tight-fitting N95 mask and eye protection will often reduce fog formation.

Neuroimaging with MRI is done once the patient arrives at radiology department. The MRI (3T Ingenia, Philips Medical Systems) protocol consists of DiffusionWeighted Image (DWI) followed by MR angiography (MRA) and Fluid-attenuated inversion recovery (FLAIR). After the third sequence, a multidisciplinary team will decide for thrombolysis with thrombectomy or thrombectomy alone which will then reduce door to decision time significantly (Table 1)

If there are positive pulmonary symptoms such as unequal breath sounda or a slight increase in temperature, we will consider low-dose chest $\mathrm{CT}$ at the same time as the CT angiography brain and neck. CT chest may aid in the diagnosis of COVID-19 (7). If there is any suspicion of ground-glass appearance on the low dose CT thorax, we will presume that the patient may have COVID-19 and will be referred to the nearest COVID-19 hospitals ${ }^{7,8}$.

If CTA is already performed in the referring hospital and a large vessel occlusion (LVO) is identifies, a special direct pathway to the angiography room is created in order to reduce the exposure to the RESQ and radiology personnel ${ }^{9,10}$.

We have a standard MRI room with ceiling mounted air conditioner. The room is neither positive nor negative pressured. HCW who transfer the patient will always be in full PPE and will be inside the MRI room during the examination, with hearing protection device. This is to reduce contamination to the MRI control room and limit the burnt rate of PPE. We covered the gantry table with plastic. However, the head coil will not be covered since it may interfere with the MRI signal, may cause claustrophobia and potentially has suffocation hazard to the patient. Terminal cleaning is mandatory after each case.

Our angiographic room is equipped with positive pressure ventilation and High-efficiency particulate air (HEPA). Due to the presence of positive pressure ventilation, the air will flow from the main angiographic suite to the next adjacent lower pressure rooms. It is important for the person/s inside the control room, scrub area and anteroom with the dual airlock system to wear a proper N95 mask. Door to the angiography room should be kept closed peri and postoperatively. Non-essential objects are removed from the angiographic room to ease post-procedure cleaning and to minimize contaminated surface areas ${ }^{2}$.

The angiographic machine especially the angiographic tubes, detectors and other are covered with designated plastics. Terminal cleaning of the room is mandatory after each case. The technologist will clean the angiographic monitor and the machine control apparatus. The cleaning process usually takes up to 1 hour.

To reduce the number of $\mathrm{HCW}$ involved during the procedure, the minimum operational $\mathrm{HCW}$ in our setting is 4 persons; they are 1 main operator (Interventional Radiologist), 1 operator assistant (Interventional Radiology trainee), 1 staff nurse and 1 radiographer. Social distancing of at least 1 meter in between team members outside the angiographic room is observed. We prefer conscious sedation with local anaesthesia, whenever possible, for our mechanical thrombectomy procedure ${ }^{11}$. Aerosol generating procedures such as high flow oxygen and self-inflating bag are not recommended due to risk of dissemination of the virus pathogen. Precaution to minimize exposure must be taken by the anaesthetic team. Further steps need to be taken such as using powered air-purifying respirator (PAPR), headbox and camera laryngoscope to ease intubation.

The standard technique of mechanical thrombectomy is applied. It is imperative to minimize the procedural time which substantially will also reduce the contact hours of the attending HCW with the patient. We dedicate an area at the anteroom with an airlock system for the operators to doff their PPEs. The HCWs are encouraged to observe each other during doffing procedure to minimize risks of contamination. The clinical waste and disposable equipments will be collected and put into biohazard labeled plastic bags ${ }^{2}$.

The patient will then be transferred to ICU with all the receiving staffs protected with PPE. It is important to reduce the time of transfer to prevent contamination to the surrounding. The patient will be transferred via a special isolated lift elevator.

In the ICU, the patient will be placed in an isolation room. All procedures will adhere to COVID-19 protocol for examinations, clinical assessments and nursing care, until their COVID-19 status is known. The swab test done earlier during admission will be traced on Day 1 of admission. Once the result of RT-PCR COVID-19 is negative; the patient will be transferred out from isolation and treated as usual patient.

If the transfer requires maintaining intubation; there is a special designated close circuit portable ventilator. The patient will be placed in the ICU. The management of the patient must adhere to COVID-19 protocol. Once the patient is stabilized, the decision to extubate will be made collectively by the respective departments. Care must be taken during extubation to minimize potential exposure to the surrounding and the HCW.

In conclusion, it is feasible to start an emergency mechanical thrombectomy service during a pandemic. The initiatives taken to ensure the safety of the healthcare worker (HCW) will not compromise patient care.

\section{Conflict of Interest}

None declared.

\section{Funding}

This manuscript received no specific grant from any funding agency/ies from the public, commercial, or notfor-profit sectors.

\section{ACKNOWLEDGEMENT}

All the authors would like to thank staff in the department of Radiology, RESQ, department of Neuromedical, department of Anaesthesia, and all HPUPM staff with the assistance producing this manuscript. 


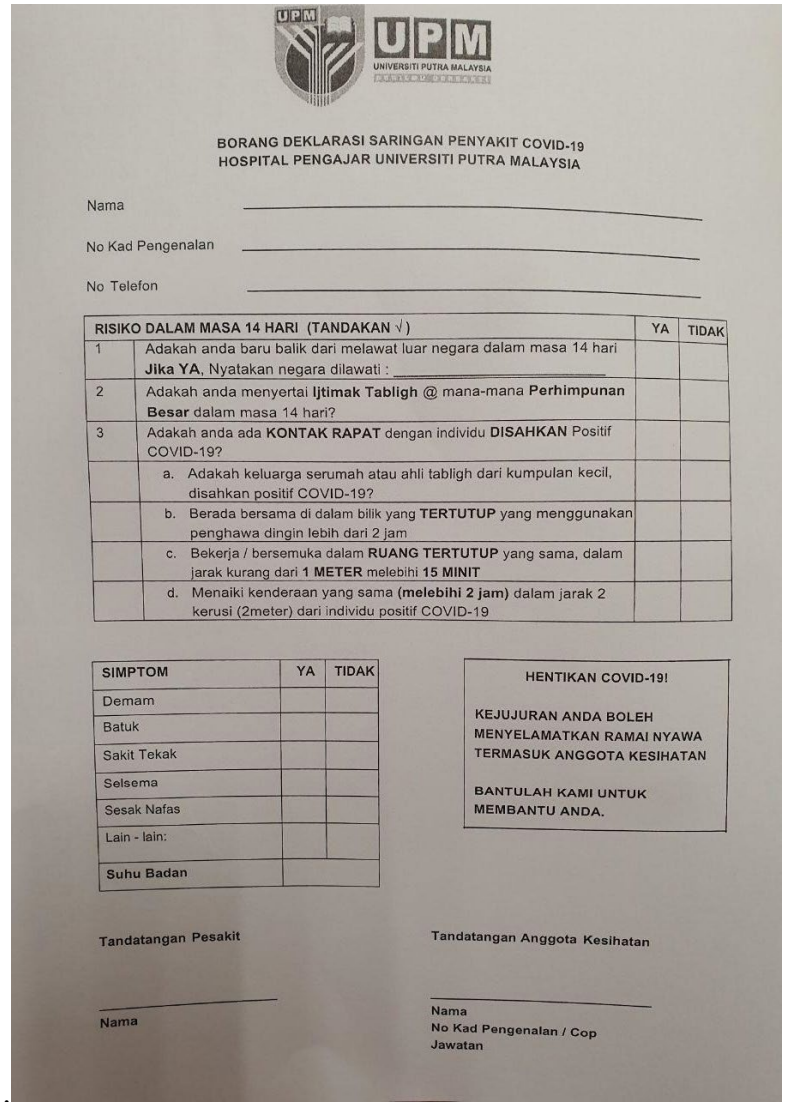

Figure 1. Declaration form to be filled and signed by the patient or next of kin

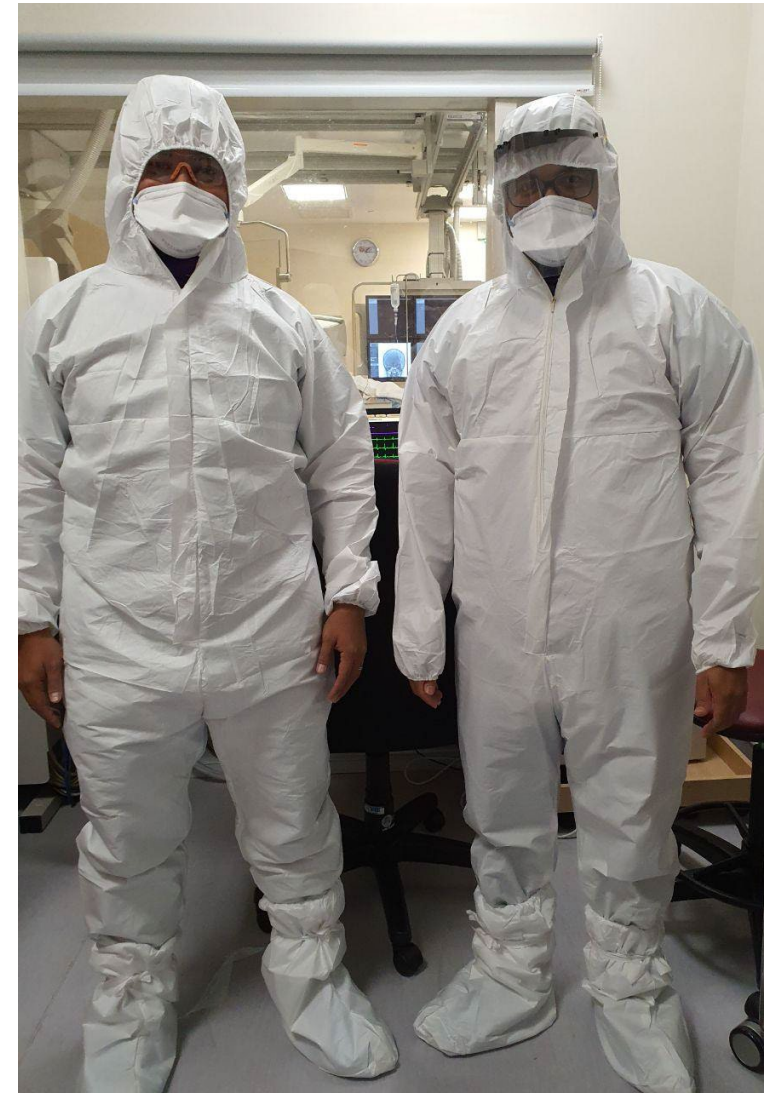

Figure 2. Full PPE adhered by the Interventional Radiologist team

Stroke code activated to alert multidisciplinary team

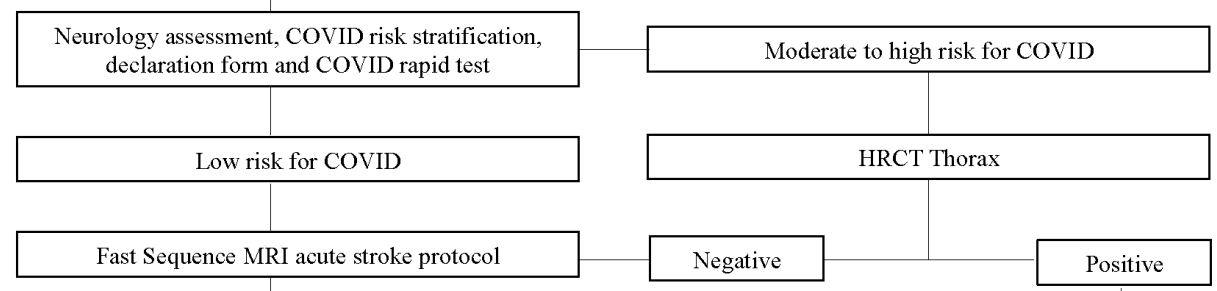

\begin{tabular}{|l|l|}
\hline $\begin{array}{l}\text { Duration } \\
\text { (min) }\end{array}$ & Sequence \\
\hline 3 & DWI \\
\hline 4 & MRA TOF \\
\hline 3 & FLAIR \\
\hline 3 & SWI \\
\hline 3 & ASL Perfusion \\
\hline
\end{tabular}

- Urgent PCR COVID Test

- Activate COVID pathway

- Multidisciplinary COVID task force case

discussion to decide on appropriate acute management
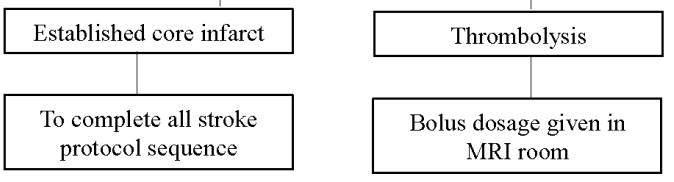

Thrombectomy

Direct transfer to angiogram suite

Table 1. Our Institutional MRI protocol and its acquisition time 


\section{REFERENCES}

1. Cucinotta D, Vanelli M. "WHO Declares COVID-19 a Pandemic.” Acta Biomed. 91(1) (2020): 157-160. 9397.

2. Nguyen TN, Abdalkader M, Jovin TG, Nogueira RG, Jadhav AP, Haussen DC, Hassan AE, Novakovic R, Sheth SA, Ortega-Gutierrez S, Panagos PD, Cordina SM, Linfante I, Mansour OY, Malik AM, Narayanan S, Masoud HE, Chou SH, Khatri R, Janardhan V Yavagal DR, Zaidat OO, Greer DM, Liebeskind DS. "Mechanical Thrombectomy in the Era of the COVID19 Pandemic: Emergency Preparedness for Neuroscience Teams: A Guidance Statement from the Society of Vascular and Interventional Neurology." Stroke 2020

3. Raja S, Patolia H, Baffoe-Bonnie A. "Calculating an institutional personal protective equipment (PPE) burn rate to project future usage patterns during the 2020 SARS-CoV-2 pandemic". Infect Control Hosp Epidemiol. 2020.

4. Oxley TJ, Mocco J, Majidi S1, Kellner CP, Shoirah H, Singh IP, De Leacy RA, Shigematsu T, Ladner TR, Yaeger KA, Skliut M, Weinberger J, Dangayach NS, Bederson JB, Tuhrim S1, Fifi JT. "Large-Vessel Stroke as a Presenting Feature of Covid-19 in the Young". N Engl J Med. 2020.

5. Khosravani H, Rajendram $P$, Notario L, Chapman MG, Menon BK. "Protected code stroke. Hyperacute stroke management during the coronavirus disease 2019 (COVID-19) pandemic." Stroke 2020.

6. Laws of Malaysia Act 342 Prevention and Control of Infectious Diseases Act 1988- Incorporating latest amendment - P.U. (A) 374/2006.
7. Adam Bernheim, Xueyan Mei, Mingqian Huang, Yang Yang, Zahi A. Fayad, Ning Zhang, Kaiyue Diao, Bin Lin, Xiqi Zhu, Kunwei Li, Shaolin Li, Hong Shan, Adam Jacobi, and Michael Chung. "Chest CT Findings in Coronavirus Disease-19 (COVID-19): Relationship to Duration of Infection". Radiology 0 (2020) :0.

8. Heng Meng,a Rui Xiong,a Ruyuan He,a Weichen Lin,a Bo Hao,a Lin Zhang,a Zilong Lu,a Xiaokang Shen,a Tao Fan,a Wenyang Jiang,a Wenbin Yang,b Tao Li,b Jun Chen,b and Geng Qinga. "CT imaging and clinical course of asymptomatic cases with COVID-19 pneumonia at admission in Wuhan, China." J Infect. 2020.

9. Mokin M, Ansari SA, McTaggart RA, Bulsara KR, Goyal M, Chen M, Fraser JF; "Indications for thrombectomy in acute ischemic stroke from emergent large vessel occlusion (ELVO): report of the SNIS Standards and Guidelines Committee. Society of NeuroInterventional Surgery." J Neurointerv Surg. 11(3) (2019):215-220.

10. Hashim, Hilwati; Hassan, Radhiana; Sharis, Syazarina; Azmin, Shahrul; Remli, Rabani; Mukari, Shahizon Azura; Yahya, Nafisah; Tan, Hui Jan; Mohamed Ibrahim, Norlinah; Ismail, Mohd Saiboon; Muda, Sobri; Sahathevan, Ramesh. "Evaluation of time-dependent pathways in an acute ischemic stroke protocol that incorporates CT perfusion: A tertiary referral center experience.” Neurology Asia 18 (4) (2013): 355-360.

11. Abou-Chebl A, Lin R, Hussain MS, Jovin TG, Levy EI, Liebeskind DS, et al. "Conscious sedation versus general anesthesia during endovascular therapy for acute anterior circulation stroke: preliminary results from a retrospective, multicenter study." Stroke. 41 (2010) :1175-1179. 